brain tumour patients treated in childhood. Acta Paediatrica Oct 2006;95:1291-1297). (Respond: Dr S Pietila, Department of Paediatrics, Tampere University Hospital, PO Box 2000, FI-33521 Tampere, Finland).

COMMENT. The cause of the osteoporosis among children treated for brain tumor is multifactorial, and includes craniospinal irradiation. Other possible factors are glucocorticoid treatment, motor handicaps, and impaired nutrition. Early identification could lead to effective therapy.

\title{
DEMYELINATING DISEASES
}

\section{CLINICAL MANIFESTATIONS OF MULTIPLE SCLEROSIS IN TAIWANESE CHILDREN}

Twenty-one patients with multiple sclerosis (MS) and onset before 18 years were treated over the past 22 years and their records retrospectively analyzed at the National Taiwan University Hospital, Taipei, and Min-Sheng General Hospital, Taoyuan, Taiwan. Fifteen were female and 6 male, mean age $12.4+/-4.5$ years. Presenting symptoms or signs in order of frequency were limb weakness $(62 \%)$, visual loss or field defect $(43 \%)$, bulbar symptoms $(33 \%)$, sensory disturbance $(29 \%)$, headache $(29 \%)$, ataxia $(19 \%)$, bowel or sphincter dysfunction (14\%), and encephalopathy or encephalitis (14\%). Multiple symptoms occurred at onset in $76 \%$. A viral prodrome, usually upper respiratory, was reported 2 weeks before onset of MS symptoms in $43 \%$.

MRI at onset showed lesions in the cerebral white matter in $72 \%$, most commonly in periventricular white matter $(56 \%)$, in basal ganglia $(33 \%)$, cerebellum $(28 \%)$, spinal cord $(28 \%)$, corpus callosum $(22 \%)$, and optic nerve $(17 \%)$. Visual evoked potentials were abnormal in $77 \%$, and a total of $62 \%$ had optic nerve involvement. Only one had opticospinal MS. Of 9 patients receiving periodic subcutaneous interferon beta-1a, $4(44 \%)$ had no relapses. The course was relapsing remitting in $86 \%$, and secondary progressive in $14 \%$. The mean interval between the first and second attack was $7.2+/-10$ months, most occurring within 12 months. Three (14\%) patients who were initially diagnosed with acute disseminated encephalomyelitis developed MS after 4 months, 2 and 6 years intervals. (Weng W-C, Yang C-C, Yu T-W et al. Multiple sclerosis with childhood onset: Report of 21 cases in Taiwan. Pediatr Neurol November 2006;35:327-334). (Respond: Dr Wang-Tso Lee, Department of Pediatrics, National Taiwan University Hospital, 7 Chung-Shan South Road, Taipei, Taiwan).

COMMENT. Compared with the West, Asian populations appear to be less susceptible to MS, suggesting a genetic racial factor in susceptibility. The concordance rate for MS among monozygotic twins is $26 \%$ compared to $2 \%$ for dizygotic twins (Ebers GC et al. N Engl J Med 1986;315:1638). A 50-fold increase in risk for daughters is noted in female MS patients (Sadovnick AD et al. Neurology 1988;38:990-991). The female to male sex ratio has increased over the past 50 years and now exceeds 3.2:1 in Canada (Orton S-M et al and Canadian Collaborative Study Group. Lancet Neurol Nov 2006;5:932-936). An environmental factor is suspected. Similar changes in sex ratio are observed in USA, Australia, and Japan. In the above Taiwan series, the female to male ratio is 2.5:1. An MS 
phenotype may be defined by its association with other autoimmune diseases; $26 \%$ index MS cases reported coexisting Hashimoto thyroiditis in $10 \%$, psoriasis $(6 \%)$, and rheumatoid arthritis (2\%). (Barcellos LF et al. Lancet Neurol Nov 2006;5:924-931).

The differentiation of ADEM and MS is difficult, and in the above report, a viral prodrome and polysymptomatic presentation, characteristic of ADEM, were common in MS. Dale RC et al (Arch Dis Child 2005;90:636-639; Ped Neur Briefs June 2005;19:47-48) found that the disseminated demyelinative lesions on MRI are cortical and in deep grey matter in ADEM and periventricular/callosal in location in MS. In another study (Mikaeloff et al. Brain 2004;127:1942-1947) the risk of MS is significantly higher with MRI callosal lesions, while basal ganglia lesions are equally frequent in ADEM and MS. The risk of relapse, and subsequent diagnosis of MS in patients initially considered ADEM is $10 \%$ in one previous study (Tenembaum et al. 2002; Ped Neur Briefs 2002;16:81).

\section{METABOLIC NEURODEGENERATIVE DISORDERS}

\section{DIAGNOSIS AND TREATMENT OF SEPIAPTERIN REDUCTASE DEFICIENCY}

The diagnosis and long-term effects of treatment of two cases of sepiapterin reductase deficiency (SRD) are reported from Service de Neuropediatrie, CHU Montpellier, France; University de Sherbrooke, Quebec, Canada; and centers in Germany and Switzerland. Patient 1, a male with first-cousin Turkish parents, presented with a prolonged afebrile seizure at 6 months of age. Progressive global delay, intermittent dystonic movements, and oculogyric crises followed. EEG, MRI, and routine metabolic tests were normal. At 18 months he had developed generalized spasticity, and by 7 years of age, symptoms showed a diurnal variation, with ability to walk in the mornings and requiring a wheelchair by afternoon. Patient 2, a female born of nonconsanguineous Caucasian parents, presented with delayed psychomotor signs at 5 months, generalized hypotonia, and oculogyric crises. At one year dystonic movements and swallowing difficulties developed. She walked at 3 years, hypotonia was replaced by spasticity, and her IQ was measured at 46 . Oculogyric crises stopped at 10 years, the head circumference remained at the $50^{\text {th }}$ percentile, and EEG and MRI were normal. A diagnosis of cerebral palsy was suspected until a CSF neurotransmitter study at 13 years of age confirmed a SRD encephalopathy. CSF sepiapterin levels were elevated, and a phenylalanine loading test showed a marked increase in phenylalanine. Plasma serotonin levels were decreased and plasma prolactin was increased. Sepiapterin reductase activity was deficient in fibroblasts from both patients, a homozygous mutation in the SPR gene was found in patient 1 , and a homozygous nonsense mutation in patient 2 . Treatment was started at 7 and 12 years of age, initially with levodopa and later, with the addition of 5hydroxytryptophan. Response was dramatic, with resolution of pyramidal signs, dystonic gait, and tremor, muscle tone becoming normal within 2 years. After 5 year follow-up, patients were in school, language and motor development were improved, mild dystonia persisted, and IQ remained subnormal. CSF sepiapterin and 5-OH-tryptophan and plasma prolactin levels, used to monitor progress, had not completely normalized. (Echenne B, Roubertie A, Assmann B et al. Sepiapterin reductase deficiency: Clinical presentation and evaluation of long-term therapy. Pediatr Neurol November 2006;35:308-313). (Respond: Dr 